\title{
PIV measurement of mainstream flow in cigarette smoke
}

\author{
Jingsong Zeng ${ }^{1, a}, \quad$ Runyu $\mathrm{Li}^{2, b}, \quad$ Liang $\mathrm{Du}^{3, \mathrm{c}}$ \\ ${ }^{1}$ Fivemountain Road, Guangzhou City, Guangdong Province, China \\ ${ }^{2}$ Pingjiang County, Yueyang City, Hunan Province, China \\ ${ }^{3}$ Xinzhou District, Wuhan City, Hubei Province, China \\ 'afezenggis@scut.edu.cn, ${ }^{\text {b270302605@qq.com }},{ }^{\text {cffeduuiang@mail.scut.edu.cn }}$
}

Keywords : PIV; mainstream smoke flow field; cigarette outlet velocity.

Abstract Based on stepping motor system and the law of fluid continuity, PIV technique was used to measure mainstream flow field in cigarette smoke directly, and the cigarette outlet velocity was obtained at different stepping motor frequencies with different suction pressure. The results show that the velocities increase with the different suction.

\section{Introduction}

Cigarette smoke is poisonous and harmful to the environment. In order to study the diffusion of cigarette smoke, PIV is used to snap the flow field of cigarette smoke. PIV is a kind of visual measurement technology used to measure the instantaneous velocity field. By using it, many scholars analyze the flow field of gas and liquid at home and abroad ${ }^{[1-2]}$. Yu Wei ${ }^{[3]}$, et al also obtain the speed field in combustion state by using PIV system to measure air diffusion velocity ; Peng Xiaoyong $^{[4]}$, et al use PIV system to measure the natural indoor smoke diffusion flow-field; By used PIV technology to analyze the smoke flow field in the wind pipe, Zeng Lei ${ }^{[5]}$, et al snap the smoke movement. Ishizukas ${ }^{[6]}$, et al use PIV to study the maximum tangential velocity of flame under vortex combustion. Shibata $\mathrm{M}^{[7]}$, et al use PIV to study flue gas flow conditions. In this paper, by using PIV measurement system, velocity characteristics of the smoke flow field are revealed with smoke particles directly as tracer particles.

\section{Experimental setup}

In this experiment, the smoke is visualized by using the self-made machine, which includes PIV system, PLC control platform, stepping motor system, smoke sleeve, fixed support, and so on. CCD and laser head belong to PIV system. The laser sheet is from the laser head, the laser-induced fluorescence technique is used in the middle of smoke sleeve and burning tip of cigarette to irradiate the measurement plane while CCD is perpendicular to the laser sheet. During the measurements, there is a one-to-one correspondence between the stepping motor frequency and suction pressure in the smoke sleeve. With the different stepping motor frequency (i.e. suction pressure), the different suction pressures lead to the different smoke flow fields even if the light sheet is same. 


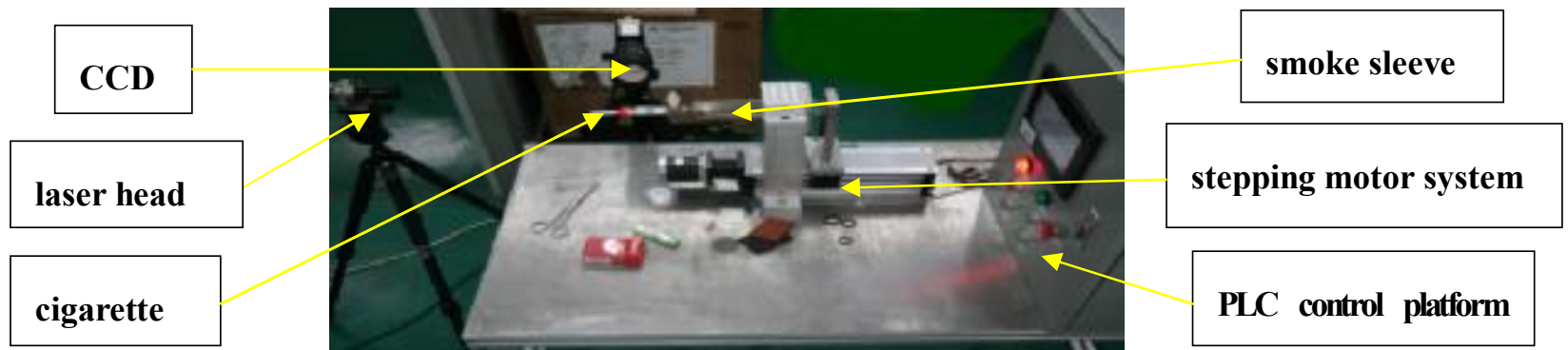

Fig. 1 The experimental equipment.

\section{Analysis and discussion}

\section{Measurement of mainstream smoke flow}

When the suction pressures are $0.01 \mathrm{KPa}, 0.05 \mathrm{KPa}, 0.13 \mathrm{KPa}, 0.21 \mathrm{KPa}, 0.29 \mathrm{KPa}$ and $0.38 \mathrm{KPa}$ respectively, the corresponding velocity flow fields of the mainstream smoke in the smoke sleeve are as shown in Fig. 2, Fig. 3, Fig. 4, Fig. 5, Fig. 6 and Fig. 7.

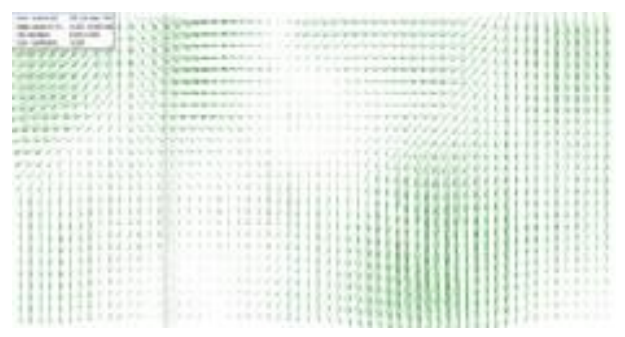

Fig.2 velocity flow field for $0.01 \mathrm{KPa}$ suction pressure

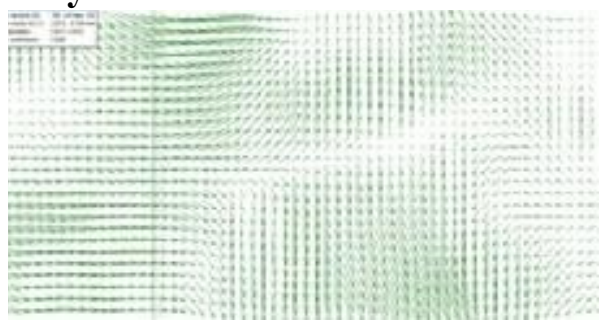

Fig.3 velocity flow field for $0.05 \mathrm{KPa}$ suction pressure

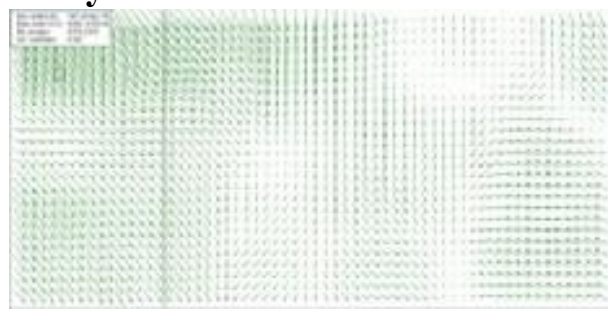

Fig.4 velocity flow field for $0.13 \mathrm{KPa}$ suction pressure

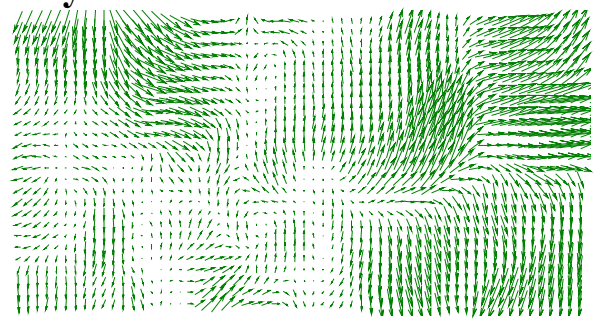

Fig.5 velocity flow field for $0.21 \mathrm{KPa}$ suction pressure 


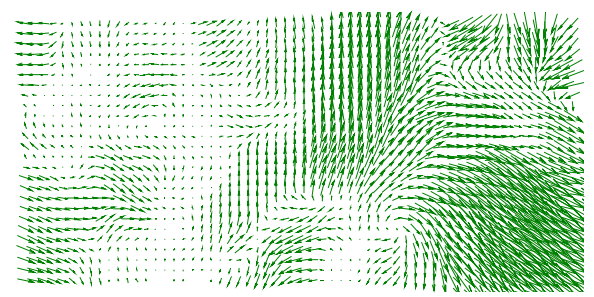

Fig. 6 velocity flow field for $0.29 \mathrm{KPa}$ suction pressure

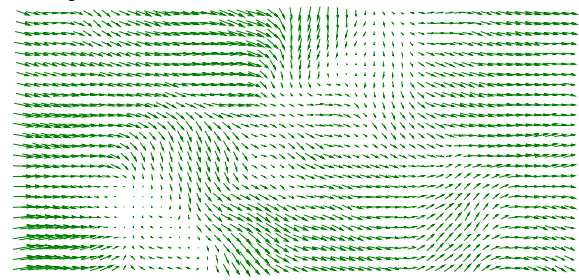

Fig.7 velocity flow field for $0.38 \mathrm{KPa}$ suction pressure

As the laser sheet is shooting the sleeve of the smoke velocity, the main stream smoke flow-fields are taken in the smoke sleeve. From the figures, it shows that there are full of all sizes vortexes in the mainstream smoke flow field, while the velocity vector flows with the suction direction. In order to obtain the cigarette exit speed, it is necessary that the smoke velocity in the sleeve is converted to cigarette export speed. As shown in Fig.8, according to the fluid continuity law Q1 = $\mathrm{Q} 2$, that is $\mathrm{u} 1 \mathrm{~A} 1=\mathrm{u} 2 \mathrm{~A} 2$. In the above express, $\mathrm{u} 1$ is the sleeve smoke velocity, $\mathrm{A}_{1}=\pi \mathrm{D}^{2} / 4$ is the sleeve area. U2 is the cigarette export speed, $A_{2}=\pi d^{2} / 4$ is the cigarette export area. Sleeve diameter $\mathrm{D}=35 \mathrm{~mm}$, outlet diameter of cigarette $\mathrm{d}=8 \mathrm{~mm}$.

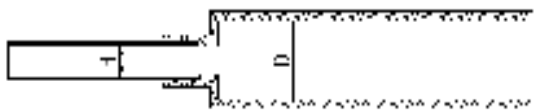

Fig.8 scheme of cigarette export and smoke sleeve

In the natural smoldering process there is no mainstream smoke, and there is no the corresponding measurement speed. The fitted curve between the outlet velocity and suction pressure, is shown in Fig.9.

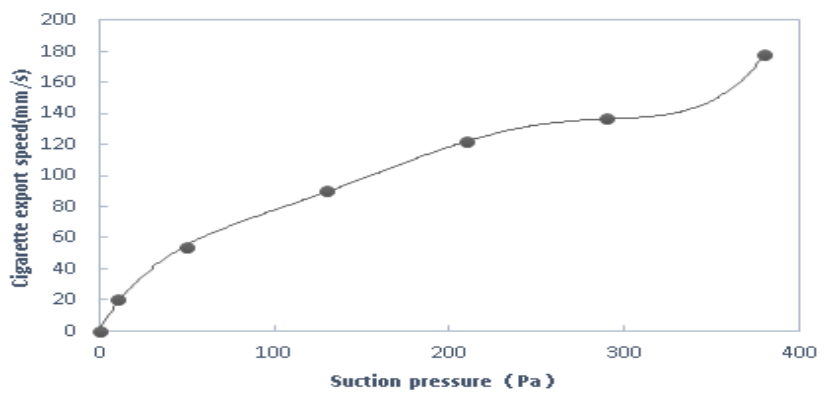

Fig.9 cigarette export speed chart

As shown in Fig.9., The fitting equation between the cigarette export velocity and suction pressure is: 


$$
y=(4 E-10) x^{5}-(4 E+0.7) x^{4}+0.0001 x^{3}-0.0191 x^{2}+1.7579 x+1.714,
$$

the correlation coefficient is 0.9995 . So the export speeds of cigarette increase with the suction pressures. According to Bernoulli equation, in the same height and medium, there are the equation (2) to be established. According to the equation, the higher suction pressure will lead to the increase of the cigarette export speed.

$$
\mathrm{P}_{1}-\mathrm{P}_{2}=\rho v_{2}^{2} / 2-\rho v_{1}^{2} / 2 \text {. }
$$

\section{Conclusion}

The velocity flow fields of the mainstream smoke end are measured by using the smoke experiment machine. The smoke is taken as the tracer particle and the different flow fields are obtained with different suction pressures. The conclusions are as follows: The higher suction pressure will lead to the increase of the cigarette export speed. The fitting equation-between the cigarette export velocity and suction pressure is:

$$
y=(4 E-10) x^{5}-(4 E+0.7) x^{4}+0.0001 x^{3}-0.0191 x^{2}+1.7579 x+1.714
$$

\section{Acknowledgements}

This work was financially supported by the Shanghai Natural Science Foundation (0666666), Innovation Program of Shanghai Municipal Education Commission (060000) and Shanghai Leading Academic Discipline Project of Shanghai Municipal Education Commission (0555555).

\section{References}

[1] Zeng, J.S., Chen, K.F, Li J., "CFD simulation of dischargers' dilution-zone in high consistency bleaching tower”, CAN. J. CHEM. ENG., 1(89), 475-483 (2011).

[2] Ceng Jinsong, Chen Kefu, CFD-based high-consistency pulp bleaching tower bottom S-type unloader, Journal of South China University of Technology, 2011 the first period of 19-23.

[3] Yu Wei, Wei Shusheng, Zhou Jianguang, Preliminary Study on Application of PIV in Combustion Flow Measurement [J], Gas Turbine Technology, 2002,15 (4): 19-41.

[4] Peng Xiaoyong, Deng Jinbo, Xie Dong, Brine experiment and PIV test for smoke diffusion in large space building fire, 2004.

[5] Zeng Lei, Zhao Lihong, particle image velocimetry (PIV) technology in the detection of tobacco flow, 2011.

[6] Satoru Ishizuka, Kiminori Koumura, Ryo Hasegawa. Enhancement of flame speed in Vortex rings of rich hydrogen / air mixtures in air [J]. Proceedings of the Combusion Institude, 2000, 28 (2): 1949-1956.

[7] Shibata M, Hirota K, Takase E, et al. Smoke flow analysis in cigarette filters. CORESTA Congress, Kyoto, 2004, Smoke Science / Product Technology Groups, abstr. PT1. 